\title{
Collagen gel droplet-embedded culture-drug sensitivity test and Ki67 expression in estrogen receptor-positive and HER2-negative breast cancer
}

\author{
KATSUNORI TOZUKA ${ }^{1}$, JUN HORIGUCHI ${ }^{1}$, DAISUKE TAKATA ${ }^{1}$, NANA ROKUTANDA ${ }^{1}$, RIN NAGAOKA ${ }^{1}$, \\ HIDEAKI TOKINIWA $^{1}$, MAMI KIKUCHI ${ }^{1}$, AYAKO SATOU ${ }^{1}$, HIROYUKI TAKEI $^{2}$ and IZUMI TAKEYOSHI $^{1}$ \\ ${ }^{1}$ Department of Thoracic and Visceral Organ Surgery, Gunma University Graduate School of Medicine, Maebashi, \\ Gunma 371-8511; ${ }^{2}$ Division of Breast Surgery, Saitama Cancer Center, Kitaadachi, Saitama 362-0806, Japan
}

Received May 15, 2012; Accepted June 12, 2012

DOI: $10.3892 / \mathrm{mco} .2012 .4$

\begin{abstract}
Anthracyclines and taxanes are standard anticancer drugs used in breast cancer chemotherapy. In general, the efficacy of chemotherapy is lower in patients with estrogen receptor (ER)-positive tumors compared to patients with ER-negative tumors. Although less chemosensitive, ER-positive disease includes a subset of patients who significantly benefit from adjuvant chemotherapy. The collagen gel droplet-embedded culture-drug sensitivity test (CD-DST) is an in vitro chemosensitivity test that has several advantages over conventional tests. The aim of the present study was to examine the correlation between CD-DST and the expression of Ki67, an indicator of tumor proliferation, to evaluate the efficacy of anthracyclines and taxanes in patients with ER-positive and human epidermal growth factor receptor 2 (HER2)-negative breast cancer. CD-DST was performed in 68 patients with ER-positive and HER2-negative breast cancer between August 2001 and November 2006. The specimens obtained during surgery were used for the CD-DST and immunohistological examination of Ki67 expression. Chemosensitivity to the anticancer drugs adriamycin (ADM), epirubicin (EPI), docetaxel (DOC) and paclitaxel (PTX) was estimated using CD-DST. Results obtained from the CD-DST showed the chemosensitivity to each anticancer drug to be ADM, 23.7\%; EPI, 75.0\%; DOC, $69.2 \%$ and PTX, $43.6 \%$. Ki67 expression was significantly higher in the group that was sensitive to DOC compared to the group that was resistant to DOC $(\mathrm{P}=0.048)$ and $\mathrm{PTX}(\mathrm{P}=0.036)$. In addition, a significant correlation was observed between a Ki67 labeling index (LI) of $>30 \%$ and chemosensitivity to PTX. In conclusion, results obtained from CD-DST and Ki67
\end{abstract}

Correspondence to: Dr Jun Horiguchi, Department of Thoracic and Visceral Organ Surgery, Gunma University Graduate School of Medicine, 3-39-22 Showa-machi, Maebashi, Gunma 371-8511, Japan E-mail: junhorigu@showa.gunma-u.ac.jp

Key words: breast cancer, chemotherapy, collagen gel dropletembedded culture-drug sensitivity test, estrogen receptor, Ki67 expression levels are able to identify a subset of patients with ER-positive and HER2-negative breast cancer who exhibit sensitivity to chemotherapy, particularly to taxane therapy.

\section{Introduction}

In operable breast cancer, anthracycline-based chemotherapy (1) and, more recently, taxane-containing regimens (2-4) have been effective in improving disease-free survival (DFS) and overall survival (OS). Breast cancer comprises at least two different entities that are defined according to estrogen receptor (ER) expression. Numerous published studies (5-10) have shown that the efficacy of chemotherapy is lower in patients with ER-positive disease compared to patients with ER-negative disease. Findings of previous studies $(1,11)$ suggested that, although less chemosensitive, ER-positive disease includes a subset of patients who significantly benefit from adjuvant chemotherapy. Although certain predictors are able to identify which ER-positive patients derive benefits from cyclophosphamide, methotrexate and fluorouracil (CMF), few data are currently available (12-14) about how to predict the efficacy of anthracyclines and either docetaxel or paclitaxel in this group.

Various biological parameters have been studied clinically for their ability to predict responses to anticancer drugs, including: i) efflux (p-glycoprotein) (15) and metabolism (CYP3A4) (16,17); ii) $\beta$-tubulin (somatic mutation of $\beta$-tubulin and change in $\beta$-tubulin isotype levels) $(18,19)$; iii) cell cycle [human epidermal growth factor receptor 2 (HER2), topII $\alpha$, Aurora-A] $(20,21)$ and iv) apoptosis (p53, BCL2 and thioredoxin) (22-24). The nuclear protein Ki67, which is present in cycling cells, is an indicator of tumor proliferation. Ki67 has shown strong prognostic effects and has been predictive of a greater response to most chemotherapies (25-29). The 2009 St. Gallen Consensus (30) recommended using markers of proliferation, such as Ki67, to determine the optimal treatment for early breast cancer.

The collagen gel droplet-embedded culture-drug sensitivity test (CD-DST) is a newly developed in vitro chemosensitivity test that has several advantages over conventional tests, such as the human tumor clonogenic assay (HTCA), the 
thymidine incorporation assay (TIA), the 3-(4,5-dimethylethiazol-2-yl)-2,5-dephenyltetrazolium bromide assay (MTT), the differential staining cytotoxicity (DiSC) assay, the histoculture drug response assay (HDRA) and the succinate dehydrogenase inhibition test (SDI). The CD-DST exhibits a high success rate in primary culture, requires a small number of cells, eliminates contamination by fibroblasts using image analysis, maintains the original growth characteristics and permits evaluation by using physiological concentrations of drugs. Feasibility of the CD-DST has been reported in breast (31), pancreatic and biliary tract (32) as well as lung cancers (33). One study (34) reported that the CD-DST is able to predict the response to chemotherapy with high accuracy in breast cancer patients.

In the present study, we examined the correlation between Ki67 expression and CD-DST to evaluate the efficacy of anthracyclines and taxanes in patients with ER-positive breast cancer.

\section{Materials and methods}

Patients. CD-DST was performed in 68 patients with ER-positive and HER2-negative invasive breast cancer who underwent surgery between August 2001 and November 2006. The surgically resected specimens were used for the CD-DST. Informed consent was obtained from each patient and the study was approved by the Ethics Committee of Gunma University.

$C D$-DST. Four anticancer drugs [adriamycin (ADM), epirubicin (EPI), docetaxel (DOC) and paclitaxel (PTX)] were used for the CD-DST. CD-DST was performed using a CD-DST kit (Primaster ${ }^{\circledR}$; Kurabou, Inc., Osaka, Japan), according to a previously described method (31). In brief, each fresh breast tumor specimen was minced with a scalpel, suspended in Hanks' balanced salt solution (HBSS), treated with Dispersion Enzyme Cocktail EZ (including 1.0\% collagenase; Kurabou, Inc.) and digested at $37^{\circ} \mathrm{C}$ for $2 \mathrm{~h}$. The dispersed cancer cells were collected by centrifugation at $250 \mathrm{x}$ g for $3 \mathrm{~min}$, filtered through a $300-\mu$ m nylon mesh, washed in HBSS, suspended in PCM-1 medium (Kurabou, Inc.) and incubated in a collagen gel-coated flask (CG-flask; Kurabou, Inc.) in a $\mathrm{CO}_{2}$ incubator at $37^{\circ} \mathrm{C}$ for $24 \mathrm{~h}$. The collagen gel in the CG-flask was dissolved in the cell dispersion enzyme EZ. Consequently, only the viable cells that adhered to the collagen gel were collected and used for sensitivity tests. Type I collagen (Cellmatrix Type CD; Nitta Gelatin, Inc., Osaka, Japan), 10X F-12 medium and reconstitution buffer were mixed together in ice water at a ratio of $8: 1: 1$. The prepared tumor cell suspension was added to the collagen solution so that the former did not exceed 1/10 of the latter solution, with the final density at $1 \times 10^{5}$ cells $/ \mathrm{ml}$. Three drops of the collagen-cell mixture ( $30 \mu \mathrm{l} / \mathrm{drop})$ were placed in each well of a 6-well multiplate on ice and allowed to gel at $37^{\circ} \mathrm{C}$ in a $\mathrm{CO}_{2}$ incubator; the final concentration was $\sim 3 \times 10^{3}$ cells/collagen gel droplet. DF medium (3 ml) (Gibco-BRL, Gaithersburg, MD, USA) was overlaid on each well $1 \mathrm{~h}$ later and incubated in a $\mathrm{CO}_{2}$ incubator at $37^{\circ} \mathrm{C}$ overnight.

To predict the response to each agent, the anticancer drugs were added (Table I). Following removal of the medium containing the anticancer drugs, each well was rinsed with $3 \mathrm{ml}$ of HBSS twice, overlaid with $4 \mathrm{ml}$ of PCM-2 (serum-free medium; Kurabou, Inc.) and incubated for a further 7 days. On the 4th day of incubation the medium was changed once. At the end of the incubation, neutral red was added to each well at a final concentration of $50 \mu \mathrm{g} / \mathrm{ml}$ and colonies in the collagen gel droplets were stained for $2 \mathrm{~h}$. Each collagen droplet was fixed with neutral formalin buffer, washed in water and quantified by image analysis. The growth rates of the control incubations were calculated as the total volume on Day $7 /$ total volume on Day 0 . In vitro sensitivity was expressed as the percentage $\mathrm{T} / \mathrm{C}$ ratio, where $\mathrm{T}$ was the total volume of the treated group and $\mathrm{C}$ was the total volume of the control group; a T/C ratio of $\leq 50 \%$ was considered to demonstrate in vitro sensitivity. For this reason, tumors were dichotomized into chemotherapysensitive and -resistant groups using a cut-off value of $50 \%$ ( $\mathrm{T} / \mathrm{C}$ ratio).

Immunohistochemical assay. Paraffin-embedded blocks from primary tumor specimens were evaluated for ER and PgR by immunohistochemistry (IHC), and for HER2 by IHC and fluorescent in situ hybridization (FISH). Tumors were considered to express ER or PgR if they showed at least $1 \%$ immunoreactive cells. Tumors were considered to be HER2positive if IHC was $3+$, or in a few cases with IHC $2+$ results, if amplified by FISH. Ki67 expression was assessed by IHC using the MIB-1 monoclonal antibody (1:200 dilution; Dako, Glostrup, Denmark). Immunostaining was performed using an automated immunostainer (Autostainer; Dako) and the results were assessed without the use of an image analysis system. The percentage of cells showing definite nuclear immunoreactivity among 2,000 invasive neoplastic cells in randomly selected, high-power (magnification, x400) fields at the periphery of the tumor was recorded and the Ki67 labeling index (LI) was calculated. Tumors were dichotomized into Ki67-high and -low tumors using the arbitrary cut-off value of $30 \%$ in this study.

Statistical analysis. Statistical analysis was performed using StatView ${ }^{\circledR}$ version 5.0. (SAS Institute, Inc.). Associations between CD-DST and Ki67 expression were assessed using Student's t-test and Chi-square or Fischer's exact tests in the case of $2 \times 2$ variables.

\section{Results}

Patient characteristics. Patient characteristics are shown in Table II. The median age was 50 years (range, 30-86 years). The proportion of patients with premenopausal status $(58.8 \%)$ was higher than that of patients with postmenopausal status. More than half of the patients had a breast tumor of $>2.0 \mathrm{~cm}$ and were positive for lymph node metastasis.

$C D$-DST. CD-DST was performed in 68 patients with ER-positive and HER2-negative invasive breast cancer. Results for each anticancer drug were as follows: ADM, 59 cases (86.8\%); EPI, 56 cases (82.4\%); DOC, 39 cases (57.3.\%) and PTX, 39 cases $(57.3 \%)$. The chemosensitivities to each anticancer drug based on the CD-CST were as follows: ADM, 23.7\%; EPI, 75.0\%; DOC, 69.2\% and PTX, 43.6\% (Fig. 1).

Correlation between Ki67 expression and clinicopathological variables. Comparisons between Ki67 expression and clinicopathological variables are shown in Table III. A significant 
Table I. Exposure conditions in CD-DST.

\begin{tabular}{lcccc}
\hline Drug & Concentration $(\mu \mathrm{g} / \mathrm{ml})$ & Exposure time $(\mathrm{h})$ & ${\text { Clinical dose }\left(\mathrm{mg} / \mathrm{m}^{2}\right)}^{\text {AUC }(\text { in vitro/human })^{\mathrm{a}}}$ \\
\hline ADM & 0.02 & 24 & 60 & 0.98 \\
EPI & 0.1 & 24 & 40 & 1.20 \\
DOC & 0.1 & 24 & 60 & 0.83 \\
PTX & 1.0 & 24 & 210 & 1.03 \\
\hline
\end{tabular}

${ }^{a}$ 1.0, same as clinical AUC.ADM, adriamycin; EPI, epirubicin; DOC, docetaxel; PTX, paclitaxel; AUC, area under the drug concentration-time curve.

Table II. Patient characteristics.

\begin{tabular}{|c|c|c|}
\hline \multirow[b]{2}{*}{ Variables } & \multicolumn{2}{|c|}{ Total patients $(\mathrm{n}=68)$} \\
\hline & No. & $\%$ \\
\hline \multicolumn{3}{|l|}{ Age (years) } \\
\hline Median & 50 & \\
\hline Range & $30-86$ & \\
\hline \multicolumn{3}{|c|}{ Menopausal status } \\
\hline Pre- & 40 & 58.8 \\
\hline Post- & 28 & 41.2 \\
\hline \multicolumn{3}{|l|}{$\mathrm{ER}(\%)$} \\
\hline$<1$ & 0 & 0 \\
\hline$\geq 1$ & 68 & 100 \\
\hline \multicolumn{3}{|l|}{$\operatorname{PgR}(\%)$} \\
\hline$<1$ & 9 & 13.2 \\
\hline$\geq 1$ & 59 & 86.8 \\
\hline \multicolumn{3}{|c|}{ Tumor size (cm) } \\
\hline$\leq 2.0$ & 15 & 22.1 \\
\hline$>2.0$ & 53 & 77.9 \\
\hline \multicolumn{3}{|c|}{ Lymph node metastasis } \\
\hline Negative & 27 & 39.7 \\
\hline Positive & 41 & 60.3 \\
\hline \multicolumn{3}{|l|}{ Nuclear grade } \\
\hline $1 / 2$ & 42 & 61.8 \\
\hline 3 & 26 & 38.2 \\
\hline \multicolumn{3}{|l|}{ Ki67 LI } \\
\hline Low $(\leq 30 \%)$ & 51 & 75.0 \\
\hline High $(>30 \%)$ & 17 & 25.0 \\
\hline \multicolumn{3}{|l|}{ LVI } \\
\hline $0 / 1$ & 47 & 69.1 \\
\hline $2 / 3$ & 21 & 30.9 \\
\hline
\end{tabular}

Pre-, premenopausal; post-, postmenopausal; ER, estrogen receptor; PgR, progesterone receptor; LI, labeling index; LVI, lymphovascular invasion.

relationship between Ki67 expression and a higher nuclear grade was observed.

Correlation between CD-DST and clinicopathological variables. Table IV shows the relationship between CD-DST

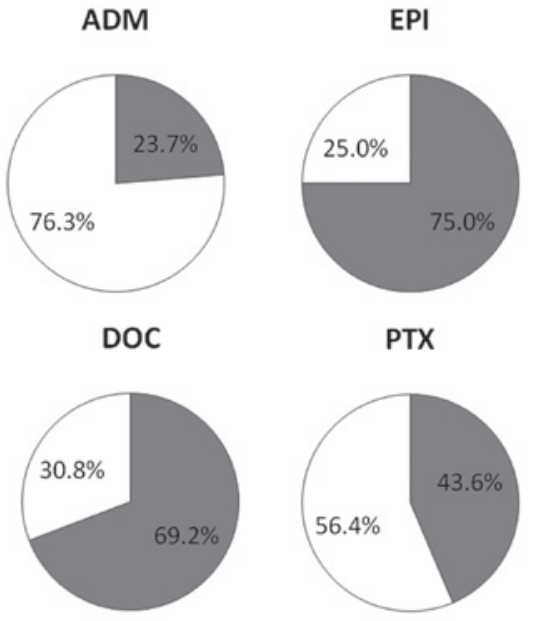

Figure 1 . Sensitivity value of each anticancer drug as determined by the collagen gel droplet-embedded culture-drug sensitivity test (CD-DST). Gray, sensitive; white, resistant. ADM, adriamycin; EPI, epirubicin; DOC, docetaxel; PTX, paclitaxel.

and clinicopathological variables in relation to the anticancer drugs. In the Chi-square test or Fischer's exact test in the case of $2 \times 2$ variables, no significant differences were evident in the relationship between the CD-DST and clinicopathological variables, with the exception of the relationship between CD-DST and Ki67 expression with PTX.

Correlation between Ki67 and CD-DST. Ki67 LI was significantly higher in the group that was sensitive to DOC compared to the group that was resistant to DOC $(\mathrm{P}=0.0480)$ and PTX ( $\mathrm{P}=0.0359)$ (Fig. 2). Regarding ADM and EPI, Ki67 LI tended to be higher in the sensitive group than in the resistant group, although there were no significant differences. Of the $11 \mathrm{Ki67-}$ high tumors, 8 (72.7\%) were diagnosed as PTX-sensitive by the CD-DST, and 9 (32.1\%) of the 28 Ki67-low tumors were diagnosed as PTX-sensitive by the CD-DST $(\mathrm{P}=0.0214)$ (Table IVD). No significant differences were observed with respect to the other anticancer drugs.

\section{Discussion}

The development of accurate predictors of chemotherapeutic responses in order to establish personalized treatment for breast cancer patients is crucial. For effective chemotherapy, the chemosensitivity testing of anticancer drugs should be performed with fresh surgical specimens or biopsy specimens 
Table III. Correlation between KI67 and clinicopathological variables.

\begin{tabular}{|c|c|c|c|}
\hline \multirow[b]{2}{*}{ Variables } & \multicolumn{3}{|c|}{ Ki67 labeling index } \\
\hline & High & Low & P-value \\
\hline \multicolumn{4}{|c|}{ Menopausal status } \\
\hline Pre- & 10 & 30 & \\
\hline Post- & 7 & 21 & \\
\hline \multicolumn{4}{|l|}{$\mathrm{ER}(\%)$} \\
\hline$<1$ & 0 & 0 & \\
\hline$\geq 1$ & 17 & 51 & \\
\hline \multicolumn{4}{|l|}{$\operatorname{PgR}(\%)$} \\
\hline$<1$ & 1 & 8 & 0.3016 \\
\hline$\geq 1$ & 16 & 43 & \\
\hline \multicolumn{4}{|c|}{ Tumor size (cm) } \\
\hline$\leq 2.0$ & 4 & 11 & 0.8659 \\
\hline$>2.0$ & 13 & 40 & \\
\hline \multicolumn{4}{|c|}{ Lymph node metastasis } \\
\hline Positive & 10 & 31 & 0.8862 \\
\hline Negative & 7 & 20 & \\
\hline \multicolumn{4}{|c|}{ Nuclear grade } \\
\hline $1 / 2$ & 6 & 36 & 0.0095 \\
\hline 3 & 11 & 15 & \\
\hline \multicolumn{4}{|l|}{ LVI } \\
\hline $0 / 1$ & 12 & 35 & 0.8795 \\
\hline $2 / 3$ & 5 & 16 & \\
\hline
\end{tabular}

Pre-, premenopausal; Post-, postmenopausal; ER, estrogen receptor; $\mathrm{PgR}$, progesterone receptor; LVI, lymphovascular invasion.

obtained from the breast cancer. Various in vitro chemosensitivity tests have been studied and developed. These include HTCA (35), TIA (36), SDI test (37), MTT assay (38), DiSC assay (39), three-dimensional agarose-based EDRA (40) and HDRA (41). However, these tests are not widely used in clinical practice for several reasons. The HTCA and TIA tests require a large sample volume; the HTCA, SDI and MTT tests have a low success rate in primary culture; and HDRA requires an extremely high concentration of drugs in the culture medium. CD-DST is a newly developed in vitro chemosensitivity test that has several advantages over conventional tests. CD-DST exhibits a high success rate in primary culture, requires a small number of cells, eliminates contamination by fibroblasts using image analysis, maintains the original growth characteristics and permits evaluation using physiological concentrations of drugs.

Takamura et al (34) demonstrated that CD-DST may be a predictive marker for chemotherapy. In their study, biopsy specimens of patients with primary breast cancers or locally recurrent breast cancers before chemotherapy were used for CD-DST and examined for sensitivity to cyclophosphamide and epirubicin (CE) therapy or DOC therapy. These authors investigated the correlation between CD-DST and clinical
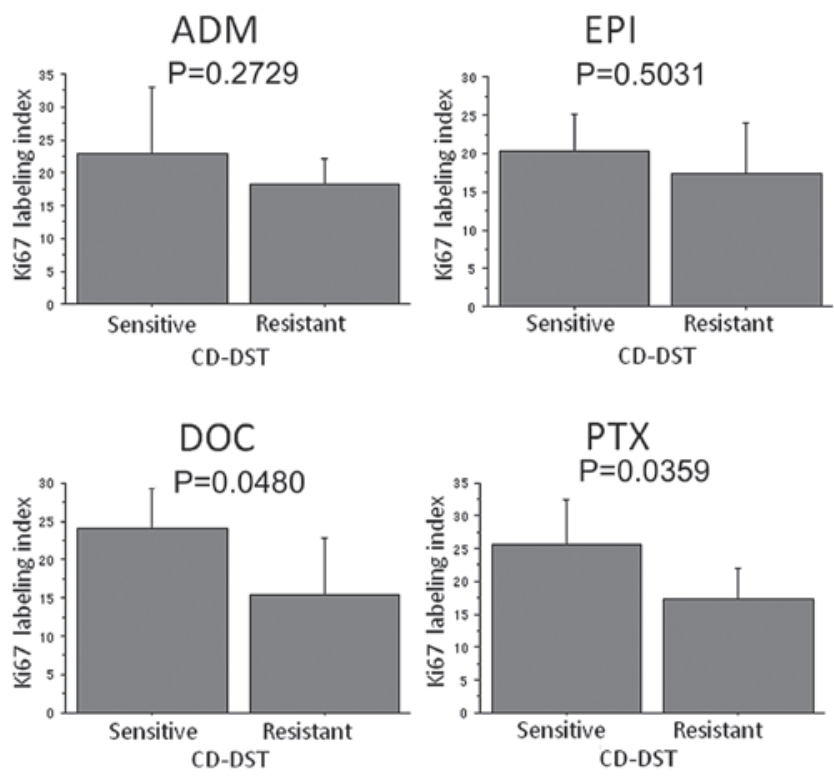

Figure 2. Ki67 labeling index and chemosensitivity to each anticancer drug. The Ki67 labeling index was significantly higher in the group sensitive to DOC compared to the group resistant to DOC $(\mathrm{P}=0.0480)$ and PTX $(\mathrm{P}=0.0359)$. ADM, adriamycin; EPI, epirubicin; DOC, docetaxel; PTX, paclitaxel.

chemotherapeutic responses and reported that CD-DST was able to predict a response to $\mathrm{CE}$ and DOC therapy with high accuracy in breast cancer patients.

We investigated chemosensitivity to ADM, EPI, DOC and PTX using CD-DST. In this study, the chemosensitivity values were found to be ADM, 23.7\%; EPI, 75.0\%; DOC, 69.2\% and PTX, 43.6\%. Yamamoto et al (42) reported that the chemosensitivity values were $30.8,53.8$ and $46.2 \%$ for ADM, DOC and PTX, respectively. Takamura et al (34) reported that the chemosensitivity values were 65.2 and $38.9 \%$ for CE and DOC, respectively. Yamamoto et al (42) investigated the sensitivity to ADM in 13 breast cancers using CD-DST. Of 13 tumors, 8 were HER2-positive, and 4 of these 8 HER2-positive tumors were sensitive to ADM. Thus, patients with HER2-positive breast cancer reportedly derive benefits from ADM (43). The sensitivity to EPI was the highest among these 4 anticancer drugs. Anthracycline-based chemotherapy may be a standard regimen even for patients with ER-positive and HER2-negative breast cancer. The sensitivity to DOC and PTX in this study was 69.2 and $43.6 \%$, respectively. The sensitivity to PTX was similar to that reported above (46.2\%). However, the sensitivity to DOC was higher than those values reported above (38.9 and 53.8\%). We selected ER-positive and HER2-negative breast cancers in this study. In general, the efficacy of chemotherapy is lower in patients with ER-positive tumors as compared with patients with ER-negative tumors $(5,6)$. Henderson et al (44) reported improved DFS as a result of adding PTX to adriamycin and cyclophosphamide (AC) for patients with ER-negative tumors, but not for those with ER-positive tumors in the adjuvant setting. Bear et al (8) showed that the pCR rate of ER-negative tumors is higher $(22.8 \%)$ than that of ER-positive tumors $(14.1 \%)$ in the neoadjuvant setting by evaluating the effect of the addition of DOC to AC in an NSABP B-27 trial. By contrast, Tham et al (45) reported that the DOC response rate of ER-positive tumors is higher (90\%) than that 
Table IV. Correlation between CD-DST and clinicopathological variables (ADM, EPI, DOC and PTX).

\begin{tabular}{|c|c|c|c|}
\hline \multirow[b]{2}{*}{ Variables } & \multicolumn{3}{|c|}{ CD-DST } \\
\hline & Sensitive & Resistant & P-value \\
\hline \multicolumn{4}{|c|}{ Menopausal status } \\
\hline Pre- & 8 & 28 & 0.7336 \\
\hline Post- & 6 & 17 & \\
\hline \multicolumn{4}{|l|}{$\operatorname{PgR}(\%)$} \\
\hline$<1$ & 1 & 7 & 0.4220 \\
\hline$\geq 1$ & 13 & 38 & \\
\hline \multicolumn{4}{|c|}{ Tumor size (cm) } \\
\hline$\leq 2.0$ & 3 & 9 & 0.9077 \\
\hline$>2.0$ & 11 & 36 & \\
\hline \multicolumn{4}{|c|}{ Lymph node metastasis } \\
\hline Positive & 11 & 23 & 0.0694 \\
\hline Negative & 3 & 22 & \\
\hline \multicolumn{4}{|c|}{ Nuclear grade } \\
\hline $1 / 2$ & 9 & 26 & 0.6651 \\
\hline 3 & 5 & 19 & \\
\hline \multicolumn{4}{|l|}{ LVI } \\
\hline $0 / 1$ & 11 & 32 & 0.5835 \\
\hline $2 / 3$ & 3 & 13 & \\
\hline \multicolumn{4}{|l|}{ Ki67 LI } \\
\hline High & 5 & 10 & 0.3113 \\
\hline Low & 9 & 35 & \\
\hline
\end{tabular}

B, EPI.

\begin{tabular}{|c|c|c|c|}
\hline \multirow[b]{2}{*}{ Variables } & \multicolumn{3}{|c|}{ CD-DST } \\
\hline & Sensitive & Resistant & P-value \\
\hline \multicolumn{4}{|c|}{ Menopausal status } \\
\hline Pre- & 28 & 7 & 0.2646 \\
\hline Post- & 14 & 7 & \\
\hline \multicolumn{4}{|l|}{$\operatorname{PgR}(\%)$} \\
\hline$<1$ & 4 & 3 & 0.2434 \\
\hline$\geq 1$ & 38 & 11 & \\
\hline \multicolumn{4}{|c|}{ Tumor size (cm) } \\
\hline$\leq 2.0$ & 10 & 2 & 0.4520 \\
\hline$>2.0$ & 32 & 12 & \\
\hline \multicolumn{4}{|c|}{ Lymph node metastasis } \\
\hline Positive & 23 & 8 & 0.8767 \\
\hline Negative & 19 & 6 & \\
\hline \multicolumn{4}{|c|}{ Nuclear grade } \\
\hline $1 / 2$ & 26 & 8 & 0.7520 \\
\hline 3 & 16 & 6 & \\
\hline \multicolumn{4}{|l|}{ LVI } \\
\hline $0 / 1$ & 31 & 10 & 0.8617 \\
\hline $2 / 3$ & 11 & 4 & \\
\hline \multicolumn{4}{|l|}{ Ki67 LI } \\
\hline High & 11 & 3 & 0.7216 \\
\hline Low & 31 & 11 & \\
\hline
\end{tabular}

Table IV. Continued.

C, DOC.

\begin{tabular}{|c|c|c|c|}
\hline \multirow[b]{2}{*}{ Variables } & \multicolumn{3}{|c|}{ CD-DST } \\
\hline & Sensitive & Resistant & P-value \\
\hline \multicolumn{4}{|c|}{ Menopausal status } \\
\hline Pre- & 17 & 6 & 0.4475 \\
\hline Post- & 10 & 6 & \\
\hline \multicolumn{4}{|l|}{$\operatorname{PgR}(\%)$} \\
\hline$<1$ & 3 & 1 & 0.7919 \\
\hline$\geq 1$ & 24 & 11 & \\
\hline \multicolumn{4}{|c|}{ Tumor size $(\mathrm{cm})$} \\
\hline$\leq 2.0$ & 7 & 3 & 0.9513 \\
\hline$>2.0$ & 20 & 9 & \\
\hline \multicolumn{4}{|c|}{ Lymph node metastasis } \\
\hline Positive & 14 & 6 & 0.9150 \\
\hline Negative & 13 & 6 & \\
\hline \multicolumn{4}{|c|}{ Nuclear grade } \\
\hline $1 / 2$ & 14 & 8 & 0.3892 \\
\hline 3 & 13 & 4 & \\
\hline \multicolumn{4}{|l|}{ LVI } \\
\hline $0 / 1$ & 20 & 9 & 0.9513 \\
\hline $2 / 3$ & 7 & 3 & \\
\hline \multicolumn{4}{|l|}{ Ki67 LI } \\
\hline High & 10 & 2 & 0.2033 \\
\hline Low & 17 & 10 & \\
\hline
\end{tabular}

\begin{tabular}{|c|c|c|c|}
\hline \multirow[b]{2}{*}{ Variables } & \multicolumn{3}{|c|}{ CD-DST } \\
\hline & Sensitive & Resistant & P-value \\
\hline \multicolumn{4}{|c|}{ Menopausal status } \\
\hline Pre- & 10 & 12 & 0.7893 \\
\hline Post- & 7 & 10 & \\
\hline \multicolumn{4}{|l|}{$\operatorname{PgR}(\%)$} \\
\hline$<1$ & 2 & 2 & 0.7894 \\
\hline$\geq 1$ & 15 & 20 & \\
\hline \multicolumn{4}{|c|}{ Tumor size (cm) } \\
\hline$\leq 2.0$ & 5 & 5 & 0.6355 \\
\hline$>2.0$ & 12 & 17 & \\
\hline \multicolumn{4}{|c|}{ Lymph node metastasis } \\
\hline Positive & 12 & 9 & 0.0652 \\
\hline Negative & 5 & 13 & \\
\hline \multicolumn{4}{|c|}{ Nuclear grade } \\
\hline $1 / 2$ & 10 & 12 & 0.7893 \\
\hline 3 & 7 & 10 & \\
\hline \multicolumn{4}{|l|}{ LVI } \\
\hline $0 / 1$ & 13 & 16 & 0.7906 \\
\hline $2 / 3$ & 4 & 6 & \\
\hline \multicolumn{4}{|l|}{ Ki67 LI } \\
\hline High & 8 & 3 & 0.0214 \\
\hline Low & 9 & 19 & \\
\hline
\end{tabular}

Pre-, premenopausal; Post-, postmenopausal; PgR, progesterone receptor; LI, labeling index; LVI, lymphovascular invasion; ADM, adriamycin; EPI, epirubicin; DOC, docetaxel; PTX, paclitaxel. 
of ER-negative tumors (50\%) in neoadjuvant chemotherapy. Results of an investigation by Learn et al (46) of the effect of adding DOC to AC suggested that ER-positive tumors are more likely to respond to DOC than are ER-negative tumors in the neoadjuvant setting. Although the predictive value of ER or HER2 status for the response to DOC or PTX remains to be established, patients with ER-positive and HER2-negative breast cancer may derive benefits from DOC rather than PTX.

The precise role and indications for chemotherapy for ER-positive breast cancer are controversial. Ki67 has been investigated as a predictive marker for chemotherapy using clinical and pathological responses as endpoints. Previous reports $(31,34)$ have demonstrated that CD-DST may predict a response to chemotherapy with high accuracy in breast cancer. We investigated the association between CD-DST and Ki67 expression in ER-positive and HER2-negative breast cancer. Higher levels of Ki67 expression tended to be sensitive to DOC and PTX. Although there is no standard pathological assessment for Ki67, the panel of experts at the St. Gallen Consensus in 2009 considered Ki67 LI to be crucial in selecting additional chemotherapy beyond endocrine therapy for patients with hormone receptor-positive breast tumors. The patients were divided into low-, intermediate- and highlyproliferating groups according to the value of Ki67 LI $(\leq 15$, $16-30 \%$ and $>30 \%$, respectively). Tumors were dichotomized into Ki67-positive and -negative tumors using the arbitrary cut-off value of $30 \%$ (Ki67 LI) in this study. We found that highly proliferating tumors were significantly more sensitive to PTX compared to the low ones.

In The Breast Cancer International Research Group (BCIRG) 001 trial, investigators stratified the hormone receptor-positive breast cancers into two subtypes: luminal A (hormone receptor-positive, HER2-negative and Ki67 $\leq 11 \%$ ) and luminal B (hormone receptor-positive, HER2-positive and/or Ki67 >11\%). Taxane-containing therapy showed a significant benefit for patients in the luminal B group, with a 3-year DFS. Thus, high levels of Ki67 expression may be a predictive marker for taxane-containing therapy (13). In the PACS01 study (14), using a cut-off value of $>20 \%$ for positive $\mathrm{Ki} 67$, the hazard ratio for relapse associated with DOC was 0.51 (95\% CI, 0.26-1.01) in patients with ER-positive/Ki67-positive tumors and 1.03 (0.69-1.55) in the ER-positive/Ki67-negative tumors. The investigators concluded that Ki67 identified a subset of DOC-sensitive, ER-positive breast cancers.

In this study, high-Ki67 tumors were found to be sensitive to taxane therapy in patients with ER-positive breast cancer. Results of recent studies $(13,14)$ have shown an association between Ki67 and sensitivity to DOC; however, no report on PTX has been published. We showed that high-Ki67 tumors were sensitive to both PTX and DOC. The association between anticancer drug sensitivity and clinicopathological variables has been evaluated, but no significant association was obtained. Although anthracyclines, particularly EPI, may be effective for patients with ER-positive breast cancer, the risk of a cardiac event is likely to be severe. If Ki67 level is high, taxanes may replace anthracyclines in patients with low cardiac function.

The possible use of Ki67 as a prognostic marker for breast cancer has been investigated (47-50). In their study, Cheang et al (48) reported that luminal breast cancers with a Ki67 level of $\geq 14 \%$ had a worse prognosis for both breast cancer recurrence and death as compared to tumors with a Ki67 level $<14 \%$. The 21-gene assay (Oncotype DX ${ }^{\mathrm{TM}}$ ) and the 70 -gene profile (Mammaprint ${ }^{\circledR}$ ) are new prognostic tools that have the potential to greatly improve risk assessment and treatment decision-making for early breast cancer $(51,52)$. Oncotype $\mathrm{DX}^{\mathrm{TM}}$, including the $\mathrm{Ki} 67$ gene, was developed specifically for patients with ER-positive breast cancer and has been shown to predict distant recurrence more accurately than classical clinicopathologic features in patients with ER-positive breast cancer and negative axillary nodes treated with adjuvant tamoxifen. The prospective validation of these assays is currently ongoing through the TAILORx and MINDACT trials $(53,54)$. CD-DST is known to predict the response to chemotherapy with high accuracy in breast cancer $(31,34)$. However, no report has been published regarding the association between CD-DST and the prognosis of breast cancer. A prospective study is therefore required to investigate the association between CD-DST and the prognosis of breast cancer. In conclusion, the CD-DST and Ki67 expression levels are capable of identifying a subset of patients who are potentially sensitive to chemotherapy.

\section{References}

1. Early Breast Cancer Trialists' Collaborative Group (EBCTCG): Effects of chemotherapy and hormonal therapy for early breast cancer on recurrence and 15-year survival: an overview of the randomized trials. Lancet 365: 1687-1717, 2005.

2. Martin M, Pienkowski T, Mackey J, et al: Breast Cancer International Research Group 001 Investigators: Adjuvant docetaxel for node-positive breast cancer. N Engl J Med 352: 2302-2313, 2005.

3. Martin M, Segui MA, Anton A, et al: Breast Cancer International Research Group 001 Investigators: Adjuvant docetaxel for nodepositive breast cancer. N Engl J Med 363: 2200-2210, 2010.

4. Sparano JA, Wang M, Martino S, et al: Weekly paclitaxel in the adjuvant treatment of breast cancer. N Engl J Med 358: 1663-1671, 2008.

5. Conforti R, Boulet T, Tomasic G, et al: Breast cancer molecular subclassification and estrogen receptor expression to predict efficacy of adjuvant anthracyclines-based chemotherapy: a biomarker study from two randomized trials. Ann Oncol 18: 1477-1483, 2007.

6. Berry DA, Cirrincione C, Henderson IC, et al: Estrogen-receptor status and outcomes of modern chemotherapy for patients with node-positive breast cancer. JAMA 295: 1658-1667, 2006.

7. Regan MM, Viale G, Mastropasqua MG, et al: Re-evaluating adjuvant breast cancer trials: assessing hormone receptor status by immunohistochemical versus extraction assays. J Natl Cancer Inst 98: 1571-1581, 2006.

8. Bear HD, Anderson S, Brown A, et al: The effect on tumor response adding sequential preoperative docetaxel to preoperative doxorubicin and cyclophosphamide: preliminary results from national surgical adjuvant breast and bowel project protocol B-27. J Clin Oncol 21: 4165-4174, 2003.

9. Stearns V, Singh B, Tsangaris T, et al: A prospective randomized pilot study to evaluate predictors of response in serial core biopsies to single agent neoadjuvant doxorubicin or paclitaxel for patients with locally advanced breast cancer. Clin Cancer Res 9: 124-133, 2003.

10. Kuerer HM, Newman LA, Smith TL, et al: Clinical course of breast cancer patients with complete pathologic primary tumor and axillary lymph node response to doxorubicin-based neoadjuvant chemotherapy. J Clin Oncol 17: 460-469, 1999.

11. Paik S, Tang G, Shak S, et al: Gene expression and benefit of chemotherapy in women with node negative, estrogen receptorpositive breast cancer. J Clin Oncol 24: 3726-3734, 2006.

12. Hayes DF, Thor AD, Dressler LG, et al: Cancer and Leukemia Group B (CALGB) Investigators: HER2 and response to paclitaxel in node-positive breast cancer. N Engl J Med 357: 1496-1506, 2007. 
13. Hugh J, Hanson J, Cheang MCU, et al: Breast cancer subtypes and response to docetaxel in node-positive breast cancer: use of an immunohistochemical definition in the BCIRG 001 trial. J Clin Oncol 27: 1168-1176, 2009.

14. Penault-Llorca F, Andre F, Sagan C, et al: Ki67 expression and docetaxel efficacy in patients with estrogen receptor-positive breast cancer. J Clin Oncol 27: 2809-2815, 2009.

15. Litman T, Druley TE, Stein WD, et al: From MDR to MXR: new understanding of multidrug resistance systems, their properties and clinical significance. Cell Mol Life Sci 58: 931-959, 2001.

16. Yamamoto N, Tamura T, Murakami H, et al: Randomized pharmacokinetic and pharmacodynamic study of docetaxel: dosing based on body-surface area compared with individualized dosing based on cytochrome P450 activity estimated using a urinary metabolite of exogenous cortisol. J Clin Oncol 23: 1061-1069, 2005.

17. Miyoshi Y, Taguchi T, Kim SJ, et al: Prediction of response to docetaxel by immunohistochemical analysis of CYP3A4 expression in human breast cancers. Breast Cancer 12: 11-15, 2005.

18. Hasegawa S, Miyoshi Y, Egawa C, et al: Mutational analysis of the classIbeta-tubulin gene in human breast cancer. Int J Cancer 101: 46-51, 2002

19. Burkhart CA, Kavallaris M and Band Horwitz S: The role of beta-tubulin isotype in resistance to antimitotic drugs. Biochim Biophys Acta 1471: 1-9, 2001

20. Coon JS, Marcus E, Gupta-Burt S, et al: Amplification and overexpression of topoisomerase II $\alpha$ predict response to anthracycline-based therapy in locally advanced breast cancer. Clin Cancer Res 8: 1061-1067, 2002.

21. Anand S, Penrhyn-Lowe S and Venkitaraman AR: AURORA-A amplification overrides the mitotic spindle assembly checkpoint, inducing resistance to taxol. Cancer Cell 3: 51-62, 2003.

22. Sjostrom J, Blomqvist C, Heikkila $\mathrm{P}$, et al: Predictive value of $\mathrm{p} 53, \mathrm{mdm}-2, \mathrm{p} 21$, and mib-1 for chemotherapy response in advanced breast cancer. Clin Cancer Res 6: 3103-3110, 2000

23. Sjostrom J, Blomqvist C, von Boguslawski K, et al: The predictive value of bcl-2, bax, bcl-xL, bag-1, fas, and fasL for chemotherapy response in advanced breast cancer. Clin Cancer Res 8: 811-816, 2002 .

24. Yokomizo A, Ono M, Nanri H, et al: Cellular levels of thioredoxin associated with drug sensitivity to cisplatin, mitomycin $\mathrm{C}$ doxorubicin, and etoposide. Cancer Res 55: 4293-4296, 1995

25. Sahin AA, Ro J, Ro JY, et al: Ki67 immunostaining in node negative stage I/II breast carcinoma. Significant correlation with prognosis. Cancer 68: 549-557, 1991.

26. Domagala W, Markiewski M, Harezga B, et al: Prognostic significance of tumor cell proliferation rate as determined by the MIB-1 antibody in breast carcinoma: its relationship with vimentin and p53 protein. Clin Cancer Res 2: 147-154, 1996.

27. Trihia H, Murray S, Price K, et al: Ki67 expression in breast carcinoma. Its association with grading systems, clinical parameters, and other prognostic factors - a surrogate marker? Cancer 97: 1321-1331, 2003.

28. Chang J, Ormerod M, Powles TJ, et al: Apoptosis and proliferation as predictors of chemotherapy response in patients with breast carcinoma. Cancer 89: 2145-2152, 2000.

29. Archer CD, Parton M, Smith IE, et al: Early changes in apoptosis and proliferation following primary chemotherapy for breast cancer. Br J Cancer 89: 1035-1041, 2003.

30. Goldhhirsch A, Ingle JN, Gelber RD, et al: Thresholds for therapies: highlights of the St Gallen international expert consensus on primary therapy early breast cancer. Ann Oncol 20: $1319-1329,2009$

31. Kobayashi H: Development of a new in vitro chemosensitivity test using collagen gel droplet embedded culture and image analysis for clinical usefulness. Recent Results Cancer Res 161: 48-61, 2003.

32. Yasuda H, Takada T, Wada K, et al: A new in-vitro drug sensitivity test (collagen-gel droplet embedded-culture drug sensitivity test) in carcinomas of pancreas and biliary tract: possible clinical utility. J Hepatobiliary Pancreat Surg 5: 261-268, 1998.

33. Higashiyama $\mathrm{M}$, Kodama $\mathrm{K}$, Yokouchi $\mathrm{H}$, et al: Immunohistochemical p53 protein status in nonsmall cell lung cancer is a promising indicator in determining in vitro chemosensitivity to some anticancer drugs. J Surg Oncol 68: 19-24, 1998
34. Takamura Y, Kobayashi H, Taguchi T, et al: Prediction of chemotherapeutic response by collagen gel droplet embedded culture-drug sensitivity test in human breast cancers. Int J Cancer 98: 450-455, 2002

35. Von Hoff DD: He's not going to talk about in vitro predictive assay again, is he? J Natl Cancer Inst 82: 96-101, 1990.

36. Kern DH, Drogemuller CR, Kennedy MC, et al: Development of miniaturized, improved nucleic acid precursor incorporation assay for chemosensitivity testing of human solid tumors. Cancer Res 45: 5436-5441, 1985.

37. Fruehauf JP and Bosanquet AG: In vitro determination of drug response: a discussion of clinical applications. Principles Prac Oncol Updates 7: 12, 1993.

38. Carmichael J, De Graff WG, Gazdar AF, et al: Evaluation of a tetrazolium-based semiautomated colorimetric assay: assessment of chemosensitivity testing. Cancer Res 47: 936-942, 1987.

39. Wilbur DW, Camacho ES, Hilliard DA, et al: Chemotherapy of non-small cell lung carcinoma guided by an in vitro drug resistance assay measuring total tumour cell kill. Br J Cancer 65: 27-32, 1992.

40. Mechetner E, Kyshtoobayeva A, Zonis S, et al: Levels of multidrug resistance (MDR1) P-glycoprotein expression by human breast cancer correlate with in vitro resistance to taxol and doxorubicin. Clin Cancer Res 4: 389-398, 1998.

41. Furukawa T, Kubota T and Hoffman RM: Clinical application of the histoculture drug response assay. Clin Cancer Res 1: 305-311, 1995.

42. Yamamoto Y, Watanabe Y, Ishida N, et al: Collagen gel dropletembedded culture drug sensitivity test in human breast cancer. Gan To Kagaku Ryoho 35: 793-796, 2008 (In Japanese).

43. Paik S, Bryant J, Park C, et al: erbB-2 and response to doxorubicin in patients with axillary lymph node-positive, hormone receptor-negative breast cancer. J Natl Cancer Inst 90: 1361-1370, 1998.

44. Henderson IC, Berry DA, Demetri GD, et al: Improved outcomes from adding sequential paclitaxel but not from escalating doxorubicin dose in an adjuvant chemotherapy regimen for patients with node-positive primary breast cancer. J Clin Oncol 21: 976-983, 2003

45. Tham YL, Gomez LF, Mohsin S, et al: Clinical response to neoadjuvant docetaxel predicts improved outcome in patients with large locally advanced breast cancers. Breast Cancer Res Treat 94: 279-284, 2005.

46. Learn PA, Yeh IT, McNutt M, et al: HER-2/neu expression as a predictor of response to neoadjuvant docetaxel in patients with operable breast carcinoma. Cancer 103: 2252-2260, 2005.

47. de Azambuja E, Cardoso F, de Castro G Jr, et al: Ki67 as prognostic marker in early breast cancer: a meta-analysis of published studies involving 12,155 patients. Br J Cancer 96: 1504-1513, 2007.

48. Cheang MC, Chia SK, Voduc D, et al: Ki67 index, HER2 status, and prognosis of patients with luminal B breast cancer. J Natl Cancer Inst 101: 736-750, 2009.

49. Stuart-Harris R, Caldas C, Pinder SE and Pharoah P: Proliferation markers and survival in early breast cancer: a systematic review and meta-analysis of 85 studies in 32,825 patients. Breast 17 : 323-334, 2008

50. Urruticoechea A, Smith IE and Dowsett M: Proliferation marker Ki-67 in early breast cancer. J Clin Oncol 23: 7212-7220, 2005.

51. Paik S, Shak S, Tang G, et al: A multigene assay to predict recurrence of tamoxifen-treated, node-negative breast cancer. N Engl J Med 351: 2817-2826, 2004.

52. van de Vijver MJ, He YD, van't Veer LJ, et al: A gene-expression signature as a predictor of survival in breast cancer. $\mathrm{N}$ Engl $\mathrm{J}$ Med 347: 1999-2009, 2002

53. Sparano JA and Paik S: Development of the 21-gene assay and its application in clinical practice and clinical trials. J Clin Oncol 26: 721-728, 2008

54. Cardoso F, van't Veer L, Rutgers E, et al: Clinical application of the 70-gene profile: the MINDACT trial. J Clin Oncol 26 $729-735,2008$ 